\title{
Learning Analytics in Higher Education: A Reflection
}

\author{
John Zilvinskis, PhD \\ Assistant Professor, Department of Student Affairs Administration \\ Binghamton University, State University of New York \\ James E. Willis, III, PhD \\ Associate Adjunct Faculty, Department of Philosophy and Religion \\ University of Indianapolis
}

The idea of learning analytics has become popularized within higher education, yet many educators are uncertain about what is entailed when implementing these technologies into practice. The following article serves as an overview to the field of learning analytics for faculty, educators for whom the expectations to use these technologies continues to increase.

We additionally argue that those who work directly with students need a functional understanding of the learning analytics landscape in order to exercise their own expertise.

\section{What are Learning Analytics?}

From IBM commercials to conversations over coffee, the term analytics has become pronounced within our culture. This trending topic is often found within higher education and can be used to describe the measurement of business and (the focus of this manuscript) student learning. Perhaps the authoritative definition of learning analytics is offered by the premier scholarly community studying the subject, the Society for Learning Analytics Research (SoLAR): "Learning analytics is the measurement, collection, analysis and reporting of data about learners and their contexts, for purposes of understanding and optimizing learning and the environments in which it occurs" (as cited in Shum \& Ferguson, 2012, p. 4). The purpose of this article is to reflect on the what, who, and how of Learning Analytics (LA) so that readers are more informed regarding how these technologies may support student learning.

In their conceptual framework of analytics in higher education, Van Barneveld, Arnold, and Campbell (2012, p. 6) relate the broad term analytics, which they define as "an overarching concept described as data-driven decision making," to differentiated types of business/academic analytics, learning analytics, and predictive analytics. In this framework, business and academic analytics are used to manage an institution, whereas learning analytics is focused on the learner and uses data to improve student success. Predictive analytics can be used at all levels within higher education and draws upon historical data to predict future changes, therefore guiding action. As the relatively new field has grown, researchers have reminded scholars that the work is about Learning and Analytics. Suthers and Verbert (2013) describe the field as a middle space between these two concepts; an intersection between broad understandings of learning and the development and the explanation of analytic processes.

Recently, Learning Tools Interoperability (LTI) technology has expanded possibilities for building in LA components into learning management systems (LMS). As a protocol which allows systems to "talk" to one another, LTI enables third-party 
vendors to build blocks that can be easily inserted in LMS architecture (IMS Global, 2018). In the past few years, a cottage industry of sorts has arisen where small programming companies build custom or one-off systems to integrate into schools' homegrown or licensed LMS systems. Within the larger LMS systems, primarily Blackboard's Learn, Instructure's Canvas, and D2L's Brightspace, a host of tools have been developed with ambient student data, with dashboards as the primary mode of data consumption. Blackboard has an analytics suite which can help schools with such tasks as "predictive analytics" or help with "assessment and accreditation" (Blackboard, 2018). Instructure's Canvas Dropout Detective is designed for faculty use to identify student performance issues which may put them at-risk for unsuccessful outcomes (Canvas, 2018). D2L's Brightspace Insights similarly uses data for "actionable opportunities" (D2L Brightspace, 2018). Common amongst these systems is the use of extant data in order to provide visual indicators of student behavior which may be problematic for successful outcomes. However, what remains ongoing is taking seemingly disparate data, combining them in compelling ways, understanding the myriad of performance data, and providing actual interventions which might have a positive effect on student outcomes.

Conceptually, LA overlaps with other academic fields such as Educational Data Mining (EDM) and the learning sciences (Rosé, Dawson, \& Drachsler, 2017). According to the Handbook of Educational Data Mining, EDM is focused on the development of methods to analyze the vast and differential data within education (Romero, Ventura, Pechenizkiy, \& Baker 2010). Despite the stronger emphasis on methodology and analysis compared to LA, the results of these methods can be used to satisfy some of the aims of learning analytics such as modeling student learning progress and improving course teaching. Siemens and Baker (2012) further distinguish between EDM and LA by arguing that the former emphasizes automated discovery, whereas the latter relies on human judgment. In their argument for collaboration between the two camps, these authors write, "Both communities have the goal of improving the quality of analysis of large-scale educational data, to support both basic research and practice in education" (p. 253). Meanwhile, learning sciences (a broad, interdisciplinary field measuring learning) can serve as a conduit between computer science and learning analytic projects, informing their design and implementation (Piety, Hickey, \& Bishop, 2014). However, the field of learning analytics is currently wrestling with the extent to which learning theory is incorporated in LA projects. Shum and Crick (2012) observe that the complexities of student learning as measured in the learning sciences have yet to be extensively captured within LA processes. Some researchers have argued that analytics developed without connection to research in the learning sciences are vulnerable to validity issues, which influence their acceptance among stakeholders (Ochoa \& Worsley, 2016).

\section{How is Learning Measured in LA?}

Many researchers have used a pass/fail binary outcome in predictive modeling to measure student success. However, actually measuring student learning in an analytic project can be difficult because defining (and thusly predicting) student learning is more complex than student success (Shum \& Crick, 2016). Furthermore, 
learning outcomes differ between courses; therefore, scaling projects derived from specific theories of learning may not work for multiple courses compared with interventions designed to improve the universal goal of student success. Despite the difficulty, scholars in the LA field have called for developers to use learning theory to guide the analysis of education data (Wise \& Shaffer, 2015).

Both researchers and the LA community have actively sought to rectify the divergence between technology and theory. For example, an entire issue of the Journal of Learning Analytics focused on the application of theory to inform the development of LA projects (Dawson, Mirriahi, \& Gašević, 2015). The issue generated some interesting studies, some of which are highlighted here. In one study, researchers used learning theory on spacing (i.e., the distribution of study times vs. the consolidation of long study sessions) to evaluate the behaviors of initiating online sessions related to Massive Open Online Course (MOOC) certification (Miyamoto, Coleman, Williams, Whitehill, Nesterko, \& Reich, 2015). Findings from this research validate their theoretical underpinnings: students who spread out their

The absence of theory in LA presents an important opportunity for faculty, who are experts on student learning, to inform the design and implementation of these projects.

study sessions were more successful. In an application of group cognition theory, researchers created a tool that graphed group dynamics by focusing on terms repeated between members, which could be used by instructors to design future group activities (Kelly, Thompson, \& Yeoman, 2015). New methods, such as discourse-centric learning analytics, can be employed to measure complex aspects of learning and educator discourse such as meaning making and language typology (e.g., specific to discipline or emotional constructs) (Knight \& Littleton, 2015).

Although this research exemplifies how learning theory can be used to guide the work of analytics, studies like this are rare. Because many LA projects are guided by computer scientists, these efforts can be agnostic regarding learning theory, as Wise and Shaffer (2015) warn their colleagues in the field, "there is a danger in falling into the trap of thinking that with sufficient data, the numbers speak for themselves. In fact, the opposite is true: with larger amounts of data, theory plays an ever-more critical role in analysis" (p. 5). In their framing of the field, Knight, Shum, and Littleton (2014) describe several pedagogical approaches found within LA, including transactional, pragmatic, and constructivist approaches. In his commentary on the afore mentioned issues of the Journal of Learning Analytics, Chen (2015) argues the relationships between theory and LA is not a "one-way bridge" in that these technologies are not only guided by theory, but they themselves can contribute to generating new theories of student learning theory and educational psychology. The absence of theory in LA presents an important opportunity for faculty, who are experts on student learning, to inform the design and implementation of these projects.

\section{What Types of Data are Used?}

The instillation of data collecting and management systems has stimulated the development of learning analytic projects by allowing for the timely analysis of data from multiple sources (Ostrow, Wang, \& Heffernan, 2017). The introduction of 
these technologies provides unprecedented depth of understanding on student behavior. For example, the data collected by LMSs such as click stream data have revolutionized the type of information collected about student academic behavior (Siemens \& Long, 2011). However, learning analytic projects are not limited to the analysis of data collected via a LMS. Researchers can now draw multiple types of data from numerous systems such as admissions (demography, orientation participation, high school GPA), financial aid (household income, aid amount, Pell eligibility, total student loans taken), academic history (prior credits earned and total credits attempted), and learning management (attendance, discussion post count, late assignments, non-substantive post count, and count of messages to instructor) (Barber \& Sharkey, 2012). These data extend beyond student information to the behavior of staff and faculty interacting with students; for example, to evaluate the use of LA software to advise students, researchers can use software log, calendar application, and survey data to measure the behaviors of academic advisors (Aguilar, Lonn, \& Teasley, 2014). Certainly, there are no shortages of data points for researchers to use when implementing LA projects; however, there are continual efforts to bridge the gap between digital behavior and the physical world.

In many ways, data collected by online behavior and informational records cast a more permanent "digital footprint" compared with traditional understandings of physical behavior (e.g., listening to lectures, talking with peers, or reading a textbook) (Siemens \& Long, 2011). However, researchers in LA recognize that digital data cannot represent the whole student and differentiate between front stage online (digital behavior recorded by a LMS), backstage online (digital behavior not captured by the institution), and backstage offline (physical behavior not recorded by the institution) (Gilmore, 2014). In response to this gap in measurement of student behavior, researchers incorporate additional tools that measure various aspects related to learning (such as dispositions not recorded online) providing further guidance in modeling student behavior (Shum \& Crick, 2012). In an effort to bridge behavior observation and analytic method, researchers have used hand-coded video data of the ways engineer students solve design problems. Machine learning is then applied to the data to discover relevant patterns of behavior and improve instruction (Worsley \& Blikstein, 2014). Advances in technology have allowed researchers to measure multimodal interaction, which includes data regarding the students' physical reactions such as heartbeat, gestures, and eye movement (Blikstein, 2013). LA project designers take advantage of the proliferation of these sources of data to design comprehensive models to measure and, in some cases, predict future student behavior.

\section{How are LA Models Refined, then Scaled?}

For any LA initiative, transitioning a working model into an analytic tool is a crucial step in scaling (i.e., the process of converting quantitative trends into actionable resources for educators working with a large student body). Updating a model can address a range of issues such as recall time, access to data, or presentation of the outcome of interest (Barber \& Sharkey, 2012). Researchers also must choose what data they decide to draw from, preferring to use sources that are historical, interpretable, and authentic (Renzel \& Klamma, 2013). When working with such large data sets, 
researchers often develop tools to categorize data via a metric of understanding. For example, data that is dense (like text data) would require extensive time to process from scratch were it to be used in modeling. However, researchers can assess and categorize dense data prior to modeling, using smaller categories as proximities (Dowell, Graesser, \& Cai, 2016). Beyond developing predictive models that are accurate or visualizations that are accessible, analytic tools need to be scrutinized and evaluated in terms of capabilities and feasibility to achieve stakeholders' goals (Arnold et al., 2014). Once a learning analytic tool has been developed, the tool must be transformed so that it can be accessible for parties associated with scaling the project: affordable for administrators, accessible for support staff, usable for faculty, and reliable (and safe) for students (Ferguson et al., 2014).

Once data sets have been identified and tools have been created, LA implementers face a new set of challenges when they try to design systems that can handle hundreds of students in real time, bringing them to scale. In a description of the learning analytics cycle, Clow (2012) described four steps of analytic processes: (1) generate data from learners, (2) analyze data from established metrics, (3) intervene to enhance learning based on metric results, and (4) evaluate the changed behavior of learners. In this conceptualization of LA, Clow argues that this feedback loop relies on increasing the audience size (scaling up) to enhance model stability while also increasing the impact of LA initiatives. Often scaling will include the use of automation and require reconceptualization of course functionality with the goal of creating systems that provide real-time feedback for faculty and students (Hickey, Kelley, \& Shen, 2014). Suggestions from scaling a 30-student to a 500-student course include an incremental approach, incorporation of design-based methodology (i.e., creating learning opportunities that explore a particular type of learning), use of formal and informal assessment of student learning, and the creation of interactive features that not only measure contextual knowledge but also conceptual and procedural components of understanding. This process leads to new sets of barriers found within institutional organization that can prevent scaling.

There are numerous challenges that project teams must meet to successfully scale LA beyond an initial research exploration. Issues can stem from disagreements between organizing units, incongruence between software, growing pains associated with automation, and data accessibility for project partners (Lonn, Aguilar, \& Teasley, 2013). Often, organizational processes can slow down the process of scaling analytic models. Planning and resources allocation often impede the implementation of learning analytics (Siemens \& Long, 2011). Lack of staff can also impede the scaling process of analytics projects. In the implementation of an academic analytic initiative, Buerk (2014) described the lack of expertise among personnel in automation, analysis, and report generation as major barriers to the project's success.

\section{Who Is Involved in LA to Support the Institutional Mission?}

Numerous types of stakeholders must be included to nurture a successful LA project (Ganley \& Hart, 2017). There are a few aspects of any project to consider when including these stakeholders, such as demands, scope, and implementation. Campbell, deBlois, and Oblinger (2007) describe aspects of a successful implementation of an 
analytic project by listing campus leaders who use data to inform their decisionmaking, staff who have expertise in analyzing data, and sufficient technology to receive and process data. In another perspective of LA program design, Clow (2012) identifies four stakeholder groups associated with LA: learners, teachers, managers, and policy makers. The author uses the dimensions of speed and scale to describe how these roles relate to project demands. For example, learners and teachers prefer real-time feedback, while managers and policy makers have the resources to expand scale. Because of the wide-range of LA projects, there is a sense of scope that can be applied to different types of analytic projects that can be found on campus. Applying the conceptual framework of analytics developed by Van Barneveld et al., (2012), the authors identify a proposed level of focus for the types of analytics: academic analytics (institution), learning analytics (department/learner), and predicative analytics (all levels). In Buerk's (2014) narrative study of implementing an academics analytic initiative, the author describes using a "top-down approach" beginning with higherlevel and department-level administrators, then evolving to include stakeholders (e.g., departments chairs, instructors, advising staff, and students). These aspects of a project, demands, scope, and implementation are dependent on institutional culture (Sharkey, 2011). Often, projects will rely heavily on those who are on the front line interacting with students: faculty and advisors.

Faculty, who observe student performance either in physical classrooms or in digital environments, are often seen as key partners for analytic projects and can be tasked with intervention when analytics predict student failure (Campbell et al., 2007). Similarly, academic advisors can be asked to incorporate analytic results into their work with students. Barber and Sharkey (2012) put the results of their model in the hands of academic advisors in their use of University of Phoenix data to predict student course completion with two goals: (a) to validate the accuracy of the predicted model and (b) to, "provide actionable information to front-line advisors in a form that can increase student success" (p. 262). In another study, Aguilar et al. (2014) measured academic advisors use of a student warning system and found surprising behavior. For example, the researchers intended for the tool to be used prior to meeting with students, but advisors often used it during their meetings, which influenced the results of the intervention. When studying the unanticipated outcomes from advisors, it is important to account for departmental culture and the training needed to implement these technologies; otherwise, academic advisors may misinterpret predictions, create stereotypes based on output from a tool, or can miscommunicate learning analytic information to students. Examples such as these articulate some of the challenges of implementing analytic projects as staff may use technology in their own unforeseen way.

A last (or perhaps first) stakeholder to consider is the student, since they can provide valuable feedback on the individual interpretation and resulting behaviors in the development of LA projects. For example, in a study using data from Cognitive Tutor software, Baker (2007) was able to identify students as on or off-task in their online behavior and recommended informing students of their classification to evoke self-monitoring among off-task students. In this case, students were directly involved in the feedback loop without interpretation from faculty or advisors. LA researchers, who are often provided with large amounts of institutional data on students without 
IRB review, frequently overlook the communal and even ethical duties of including student voices in the development of these projects (Willis, Slade, \& Prinsloo, 2016). Often, researchers will develop LA projects with a focus on creating tools with high predictability rates without consideration for how they will influence student behaviors; instead, students should be seen as valuable partners when trying to design tools that can be integrated into their experience.

From government and institutional policy makers, campus administrators, faculty, advisors, and students, LA projects challenge organizational bodies to collaborate. Researchers who work to implement LA projects have also considered the broader contribution of these projects to support the mission of an institution. In their case for the value of analytics for higher education, Siemens and Long (2014) describe the ways these technologies can enhance the decision-making of institutional leaders. Specifically, the authors identify the power of analytics to transform pedagogy by improving understanding of student learning, enhance sense-making of complex topics (e.g., social networks), and relate faculty productivity with institutional outcomes. Furthermore, learning analytics as a system offers feedback that can enhance the decision-making of stakeholders at multiple levels (e.g., faculty designing pedagogy, administrators resourcing learning initiatives, policymakers setting a learning and outcome agenda) (Shum \& Crick 2016). From a fiscal standpoint, the implementation of LA presents benefits that are within higher education interests, such as business advantages for successfully educating students and meeting customer expectations of personalized digital services (Kay, Korn, \& Oppenheim, 2012).

Although the ways in which analytics can enhance institutional decision making is clearly noted, successful LA projects require careful consideration and alignment with institutional values. As with any academic initiative, LA projects must comport with institutional history, students served, organizational structure, and overall needs (Sharkey, 2011). As reflected in the section regarding the barriers to scaling, LA projects require financial resources, socio-cultural support, and pedagogical anchoring (Arnold et. al, 2014). Therefore, LA project team members need to make a clear case on the ways LA can be used to further campus goals, which includes a careful consideration of issues related to the ethics surrounding this type of work along with an understanding of the criticisms of this field.

\section{What are the Larger Implications for LA Projects?}

Reflecting on LA would be incomplete without the promotion of caution when designing these projects. One of the criticisms of the current work in LA is that it is too technologically deterministic or, to put it another way, LA implementation is established in such a way that technology dictates educational practice (Knight, Buckingham Shum, \& Littleton, 2014). Although the benefits of LA for higher education institutions have been described in this manuscript, the use of this tool presents ethical obligations for managers of these projects, particularly in the areas of data interpretation, student privacy, and storage of data (Slade \& Prinsloo, 2013). In addition, there are several ethical concerns that institutional stakeholders need to consider when implementing analytics related to distribution of resources and the profiling and tracking of students (Willis, Campbell, \& Pistilli, 2013). Furthermore, 
specific issues of ethical consideration surrounding LA projects lend themselves to principles within the academy that affect all levels of participation (administration, faculty, students) such as transparency, accountability, and assessment (Pardo \& Siemens, 2014). Though ethical approaches to learning analytics tend to offer frameworks for considering the pertinent issues, what is important from the project level is the possibility of considering how consequences, intended and unintended, affect all stakeholders.

\section{Opportunities Ahead}

As described in this essay, bringing learning analytics into practice requires collaboration among stakeholders who arrive at these projects with their own expertise and values. There are several camps responsible for bringing these projects from ideas to realities: computer scientists, institutional research staff, end users (such as academic advisors or faculty), and campus administrators (Zilvinskis \& Borden, 2017). These partnerships can present opportunities for collaboration across camps represented by distinct domains, such as the academic domain (experimentation and software development), the business domain (assessing costs associated with supporting students who struggle), and the practitioner domain (skeptical toward various processes involved in LA). When these partnerships get together, it can be the case that the voice of the practitioner is muted over the promise proposed within the academic domain and the urgency vocalized by the business domain (Buyarski, Murray, \& Torstrick, 2017).

However, it is important to remind the readership of this publication that educators play an important role in the development of these technologies. Faculty, academic advisors, and student affairs educators are experts in student learning and are uniquely qualified to inform the implementation of learning analytics into interventions that work, reminding developers and administrators the original goals of these projects. Student success, then, is dependent on those who work directly with students exercising their own expertise in the ever-changing environment of LA. Because of this, educators are not limited to the role of informing or educating developers and administrators, instead they should be the partners demanding better tools to support student learning, asking for LA that can conform to their teaching style and philosophy of learning.

There is an opportunity ahead for educators to invite developers and administrators into educational spaces to improve student outcomes. The implications of these technologies include the ability to predict student success and learning in a course so that educators may create more developmental and inclusive environments, enhancing the opportunity of success. LA can also be used beyond the course to enhance understanding of student learning within the curriculum. Furthermore, the use of LA presents several funding and research opportunities for educators to gain the resources needed to make these tools work. Although learning analytics is

...educators are not limited to the role of informing or educating developers and administrators, instead they should be the partners demanding better tools to support student learning, asking for LA that can conform to their teaching style and philosophy of learning. 
a burgeoning field, there has been some research performed on the design of these tools; however, what is needed are more studies of academic cultures that have successfully implemented these technologies and research on how to best collaborate with faculty, who have the motivation to improve student learning but may struggle with the time or expertise to implement learning analytics. Faculty can set the pace by insisting on LA and showcasing the possibility of these tools to enhance the experience of the students served.

\section{References}

Aguilar, S., Lonn, S., \& Teasley, S. D. (2014, March). Perceptions and use of an early warning system during a higher education transition program. Paper presented at the Fourth International Conference on Learning Analytics and Knowledge, Indianapolis, IN.

Arnold, K. E., Lynch, G., Huston, D., Wong, L., Jorn, L., \& Olsen, C. W. (2014, March). Building institutional capacities and competencies for systemic learning analytics initiatives. Paper presented at the Fourth International Conference on Learning Analytics and Knowledge, Indianapolis, IN.

Barber, R., \& Sharkey, M. (2012, April). Course correction: Using analytics to predict course success. Paper presented at the Second International Conference on Learning Analytics and Knowledge, Vancouver, BC, Canada.

Baker, R. S. (2007, April). Modeling and understanding students' off-task behavior in intelligent tutoring systems. Paper presented at the SIGCHI Conference on human Factors in Computing Systems, San Jose, CA.

Blackboard. (2018). Increase student success with learning analytics. Retrieved from https://www.blackboard.com/education -analytics/index.html
Blikstein, P. (2013, April). Multimodal learning analytics. Paper presented at the Third International Conference on Learning Analytics and Knowledge, Leuven, Belgium.

Buyarski, C., Murray, J., \& Torstrick, R. (2017). Learning analytics across a statewide system. New Directions for Higher Education, 2017(179), 33-42. https://doi.org/10.1002/he.20241

Campbell, J. P., DeBlois, P. B., \& Oblinger, D. G. (2007). Academic analytics: A new tool for a new era. EDUCASE Review 42(4), 40-42. Retrieved from

http://search.ebscohost.com/login.aspx? direct $=$ true $\&$ AuthType $=$ sso $\& \mathrm{db}=$ eric $\& A$ $\mathrm{N}=\mathrm{EJ} 769402 \&$ site $=$ eds-live\&custid $=083$ 900

Canvas (2018). Dropout Detective information for faculty. Retrieved from https://community.canvaslms.com/docs /DOC-9536-dropout-detectiveinformation-for-faculty

Clow, D. (2012, April). The learning analytics cycle: Closing the loop effectively. Paper presented at the Second International Conference on Learning Analytics and Knowledge, Vancouver, BC, Canada. 
D2L Brightspace (2018). Brightspace Insights. Retrieved from https://www.d2l.com/products/insights /

Dawson, S., Mirriahi, N., \& Gašević, D. (2015). Importance of theory in learning analytics in formal and workplace settings. Journal of Learning Analytics, 2(2), 1-4.

Dowell, N. M., Graesser, A. C., \& Cai, Z. (2016). Language and discourse analysis with Coh-Metrix: Applications from educational material to learning environments at scale. Journal of Learning Analytics, 3(3), 72-95.

Ferguson, R., Clow, D., Macfadyen, L., Essa, A., Dawson, S., \& Alexander, S. (2014, March). Setting learning analytics in context: Overcoming the barriers to largescale adoption. Paper presented at the Fourth International Conference on Learning Analytics and Knowledge, Indianapolis, IN.

Ganley, C. M., \& Hart, S. A. (2017). Shape of educational data: Interdisciplinary perspectives. Journal of Learning Analytics, 4(2), 6-11.

Gilmore, D. (2014). Goffman's front stage and backstage behaviors in online education. Journal of Learning Analytics, 1(3), 187-190.

Hickey, D. T., Kelley, T. A., \& Shen, X. (2014, March). Small to big before massive: Scaling up participatory learning analytics. Paper presented at the Fourth International Conference on Learning Analytics and Knowledge, Indianapolis, IN.
IMS Global. (2018). Learning tools interoperability: LTI v.3 and LTI advantage. Retrieved from https://www.imsglobal.org/activity/lear ning-tools-interoperability

Kay, D., Korn, N., \& Oppenheim, C. (2012). Legal, risk and ethical aspects of analytics in higher education. CETIS Analytics Series, 1(6), 1-30.

Kelly, N., Thompson, K., \& Yeoman, P. (2015). Theory-led design of instruments and representations in learning analytics: Developing a novel tool for orchestration of online collaborative learning. Journal of Learning Analytics, 2(2), 14-42.

Knight, S., Shum, S. B., \& Littleton, K. (2014). Epistemology, assessment, pedagogy: where learning meets analytics in the middle space. Journal of Learning Analytics, 1(2), 23-47.

Knight, S., \& Littleton, K. (2015). Discourse-centric learning analytics: Mapping the terrain. Journal of Learning Analytics, 2(1), 185-209.

Lonn, S., Aguilar, S., \& Teasley, S. D. (2013, April). Issues, challenges, and lessons learned when scaling up a learning analytics intervention. Paper presented at the Third International Conference on Learning Analytics and Knowledge, Leuven, Belgium.

Miyamoto, Y. R., Coleman, C. A., Williams, J. J., Whitehill, J., Nesterko, S. O., \& Reich, J. (2015). Beyond time-ontask: The relationship between spaced study and certification in MOOCs. Journal of Learning Analytics, 2(2), 47-69. 
Ostrow, K. S., Wang, Y., \& Heffernan, N. T. (2017). How flexible is your data? A comparative analysis of scoring methodologies across learning platforms in the context of group differentiation. Journal of Learning Analytics, 4(2), 91-112.

Pardo, A., \& Siemens, G. (2014). Ethical and privacy principles for learning analytics. British Journal of Educational Technology, 45(3), 438-450.

Piety, P. J., Hickey, D. T., \& Bishop, M. J. (2014, March). Educational data sciences: Framing emergent practices for analytics of learning, organizations, and systems. Paper presented at the Fourth International Conference on Learning Analytics and Knowledge, Indianapolis, IN.

Renzel, D., \& Klamma, R. (2013, April). From micro to macro: Analyzing activity in the ROLE sandbox. Paper presented at the Third International Conference on Learning Analytics and Knowledge, Leuven, Belgium.

Romero, C., Ventura, S., Pechenizkiy, M., \& Baker, R. S. (Eds.). (2010). Handbook of educational data mining. CRC Press.

Rosé, C. P., Dawson, S., \& Drachsler, H. (2017). LAK16 Editorial. Journal of Learning Analytics, 4(1), 3-5.

Siemens, G. (2012, April). Learning analytics: envisioning a research discipline and a domain of practice. Paper presented at the Second International Conference on Learning Analytics and Knowledge, Vancouver, BC, Canada.
Siemens, G., \& d Baker, R. S. (2012, April). Learning analytics and educational data mining: towards communication and collaboration. Paper presented at the Second International Conference on Learning Analytics and Knowledge, Vancouver, BC, Canada.

Siemens, G., \& Long, P. (2011). Penetrating the fog: Analytics in learning and education. EDUCAUSE Review, 46(5), 30-32.

Slade, S., \& Prinsloo, P. (2013). Learning analytics ethical issues and dilemmas. American Behavioral Scientist, 57(10), 1510-1529.

Sharkey, M. (2011, February). Academic analytics landscape at the University of Phoenix. Paper presented at the Second International Conference on Learning Analytics and Knowledge, Banff, Alberta, Canada.

Shum, S. B., \& Crick, R. D. (2012, April). Learning dispositions and transferable competencies: pedagogy, modelling and learning analytics. Paper presented at the Second International Conference on Learning Analytics and Knowledge, Vancouver, BC, Canada.

Shum, S. B., \& Crick, R. D. (2016). Learning analytics for 21st century competencies. Journal of Learning Analytics, 3(2), 6-21.

Shum, S. B., \& Ferguson, R. (2012). Social learning analytics. Educational Technology \& Society, 15(3), 3-26.

Suthers, D., \& Verbert, K. (2013, April). Learning analytics as a middle space. Paper presented at the Third International Conference on Learning Analytics and Knowledge, Leuven, Belgium. 
Van Barneveld, A., Arnold, K. E., \& Campbell, J. P. (2012). Analytics in higher education: Establishing a common language. EDUCAUSE Learning Initiative, 1, 1-11.

Willis, III, J. E., Campbell, J. P., \& Pistilli, M. D. (2013). Ethics, big data, and analytics: A model for application. EDUCAUSE Review Online.

Willis, III, J. E., Slade, S., \& Prinsloo, P. (2016). Ethical oversight of student data in learning analytics: A typology derived from a cross-continental, crossinstitutional perspective. Educational Technology Research and Development, 64(5), 881-901.
Wise, A. F., \& Shaffer, D. W. (2015). Why theory matters more than ever in the age of big data. Journal of Learning Analytics, 2(2), 5-13.

Worsley, M., \& Blikstein, P. (2014). Analyzing engineering design through the lens of computation. Journal of Learning Analytics, 1(2), 151-186.

Zilvinskis, J., \& Borden, V. M. H. (2017).

Concluding thoughts. New Directions for Higher Education, (179), 103. https://doi.org/10.1002/he.20247

Dr. John Zilvinskis is an Assistant Professor of Student Affairs Administration at Binghamton University - State University of New York (SUNY). His research has been published in Research in Higher Education, The Review of Higher Education and the Journal of Diversity in Higher Education. In addition, Dr. Zilvinskis co-edited a volume of New Directions for Higher Education on the topic of learning analytics and he is a two-time winner of the Association for Institutional Research's Charles F. Elton Best Paper Award.

James Willis, III, PhD teaches in the Philosophy and Religion Department at the University of Indianapolis. His research and writing includes work in learning analytics, student success, open digital badges, and the philosophy of religion. He has been published in journals like the Journal of Adolescent \& Adult Literacy, New Directions for Higher Education, the International Journal of Public Administration, and Educause Review amongst others. 